\title{
A NEW METHOD OF IMAGE ANALYSIS OF FLUORESCEIN ANGIOGRAPHY APPLIED TO AGE-RELATED MACULAR DEGENERATION
}

\author{
J. SAITO, S. T. D. ROXBURGH, D. SUTTON and A. ELLINGFORD \\ Dundee
}

\begin{abstract}
SUMMARY
Quantitative analysis of retinal and choroidal abnormalities using current photographic techniques is complex and laborious. Digital image analysis techniques using the scanning laser ophthalmoscope overcome many of the problems with present techniques and allow reliable quantitation. A prerequisite of quantitation is accurate image acquisition and registration. The authors describe a reliable method of image analysis and apply it to the quantitation of hyperfluorescence in scanning laser fluorescein angiograms of different forms of age-related macular degeneration. Retrospective analysis of scanning laser fluorescein angiograms obtained using a standardised technique was undertaken. Eighty-six angiograms from patients with agerelated macular degeneration were analysed and categorised as dry maculopathy, geographic atrophy of the retinal pigment epithelium, retinal pigment epithelial detachment (PED) or subretinal neovascularisation (SRNV). Fluorescein characteristics of both SRNV and PED showed a characteristic pattern of fluorescence. The advantages and disadvantages of the technique are discussed.
\end{abstract}

The scanning laser ophthalmoscope (SLO) is a relatively new instrument which can be used to provide images of the fundus oculi that are not available using conventional imaging techniques such as the traditional fundus camera. ${ }^{1-4}$ The images obtained with the SLO are of high quality and contrast, light levels are lower and therefore better tolerated by the patient, illumination is uniform, and dilatation of the pupil is not as necessary. The method of data collection leads naturally to digitisation and quantitative techniques. ${ }^{5-7}$

Use of the SLO to perform fluorescein angiogra-

From: Departments of Ophthalmology and Medical Physics, University of Dundee, Dundee, UK.

Correspondence to: Dr S. T. D. Roxburgh, Consultant Ophthalmologist, Ninewells Hospital and Medical School,

Dundee, DD1 9SY, UK. phy has a number of advantages over fluorescein angiography using the conventional fundus camera. For example, the wavelength of argon laser light matches the peak absorption of fluorescein. Importantly the acquisition of data, no longer limited by the repetition rate of the electronic flash, is constrained solely by the scanning rate of the ophthalmoscope. As a result, it is possible to acquire high-resolution images 25 times per second. One result of this increase in frame rate is the greater scope for the investigation of dynamic sequences of events.

There is a need to quantify images of retinal pathology in order accurately to assess and follow the natural progression of disease processes and to monitor any treatment response such as laser therapy. Quantitative analysis of retinal and choroidal abnormalities using current photographic techniques is laborious and unreliable. Digital image analysis techniques using the SLO overcome many of these problems and have great potential for providing reliable quantitation. However, a pre: requisite for quantitation is accurate automated image acquisition followed by robust image registration to compensate for eye movement during the angiogram. Following registration, there are several approaches to quantitation. A relatively simple method is to investigate the uptake of fluorescein in various areas of interest during angiography. We describe a reliable method of image analysis and demonstrate its application to the quantitation of hyperfluorescence in scanning laser fluorescein angiograms of different forms of age-related macular degeneration.

\section{METHOD}

\section{Equipment}

The system used in this project is illustrated in Fig. 1. The core of the system is the scanning laser ophthalmoscope (model SLO101, Rodenstock). It 


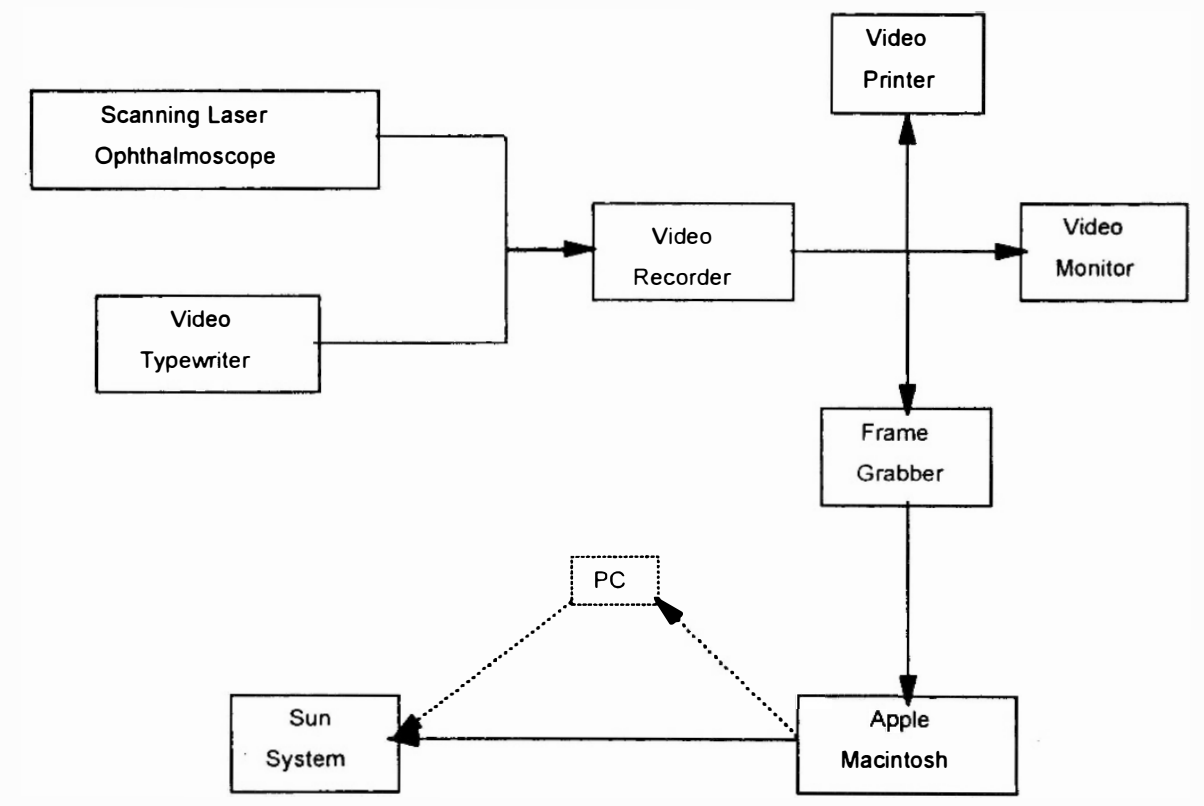

Fig. 1. Block diagram of data acquisition and processing set-up.

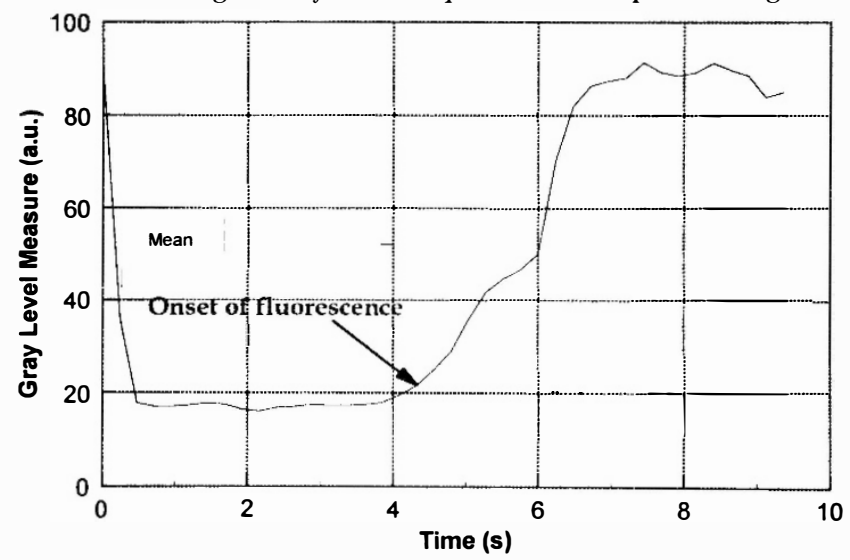

Fig. 2. Fluorescence of whole frame measured with time. The onset of fluorescence was defined as the point at which the mean grey level in two consecutive frames increased by $20 \%$.

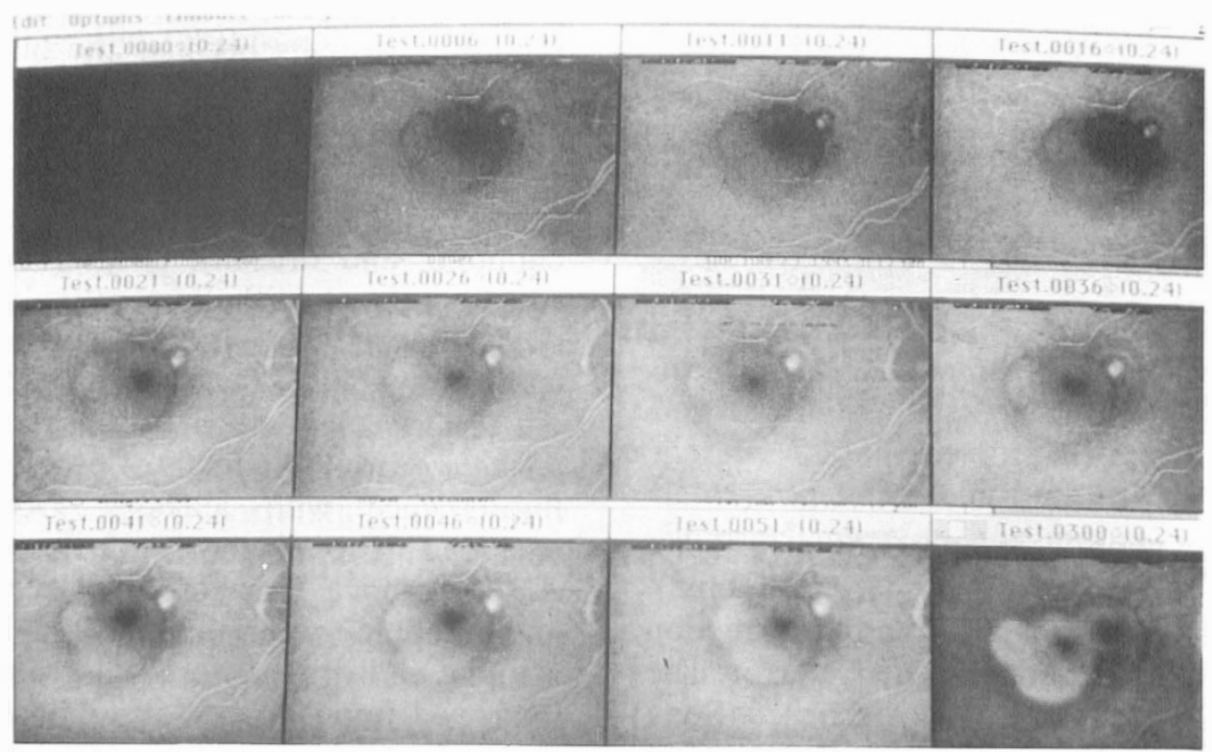

Fig. 3. A typical example of the 12 images selected for study. Images were captured at the onset of fluorescence, at 5 second intervals thereafter to 1 minute, and a late image at 5 minutes. 

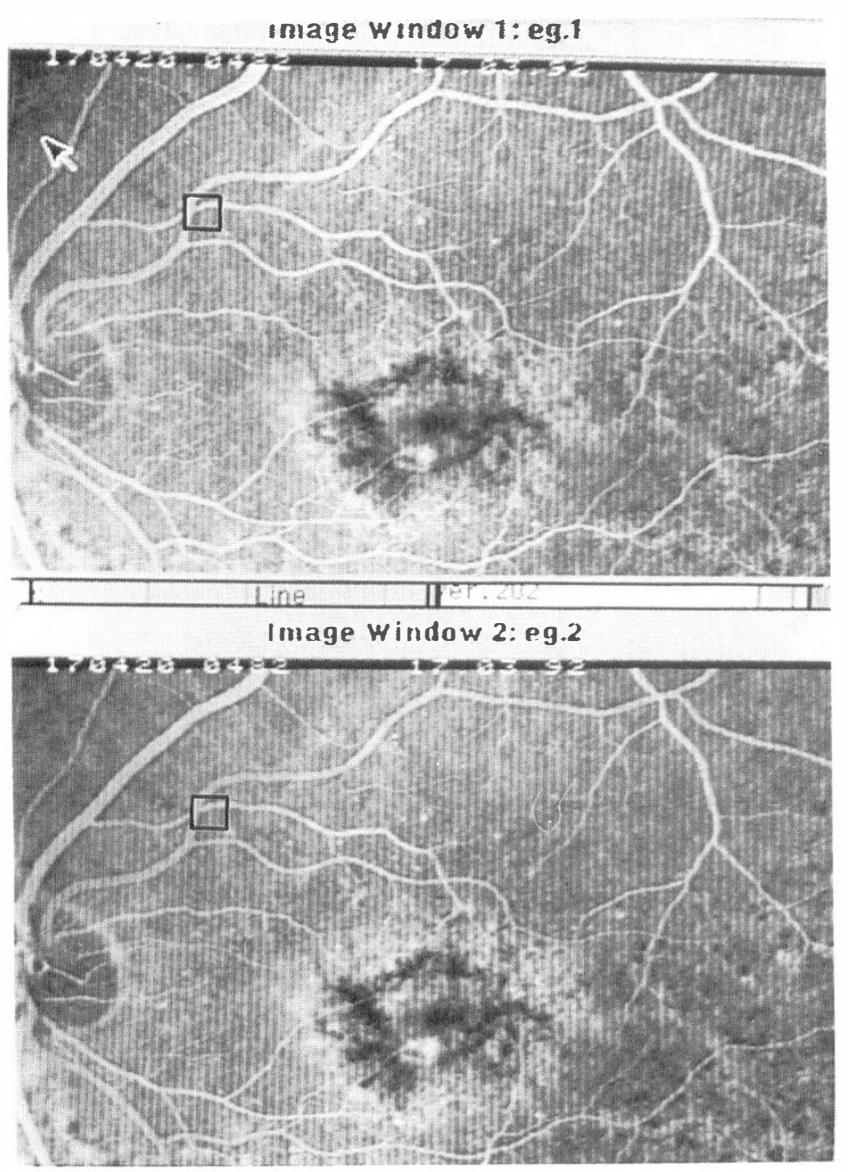

Fig. 4. A typical placement of a registration template in two sequential images.

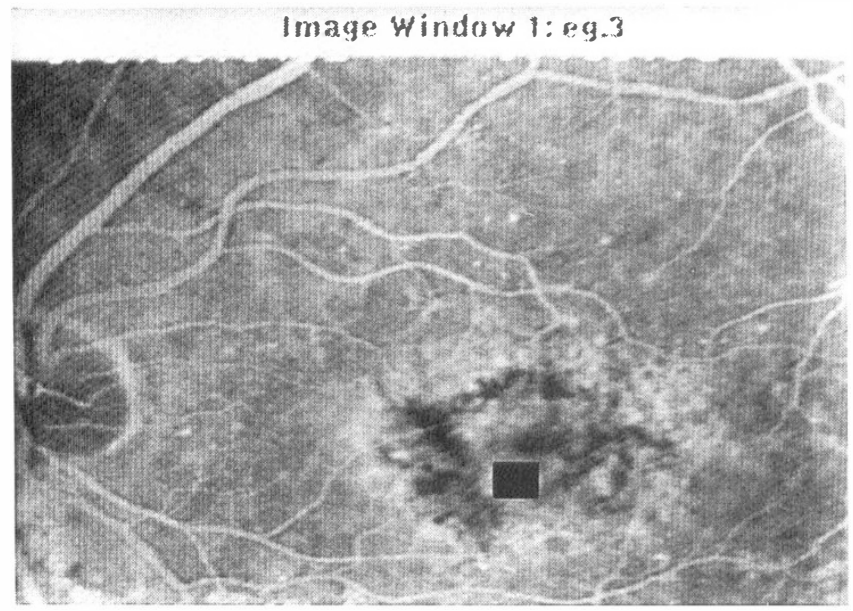

Fig. 5. The image analysis window positioned over the point of maximal fluorescence.

provides a helium-neon laser operating at $633 \mathrm{~nm}$ and an argon laser operating at $488 \mathrm{~nm}$ (blue). Integrated into the system is a refractive correction to compensate for the patient's refractive error. The video equipment consists of a video recorder (Sony VD 5800 PS), a video typewriter, a monitor, and a video printer (Sony UP 811) operating at $50 \mathrm{~Hz}$ scan rate.
In all cases the results of the SLO examination are stored on videotape and all images are marked with the patient identification number. If required for case records, video images can be printed on the thermographic video printer. Attached to the video equipment is an Apple Macintosh (model Quadra 700) fitted with a data translation frame grabber board $(769 \times 512$ pixel, 8 bit depth $)$. The grabber board is currently operated using Image 1.49 public domain software which allows some basic image processing to be performed. However, to facilitate more sophisticated image processing, images are transferred to a Sun clone (Micro Muse) computer running the Visilog imaging processing package. This package has a plethora of in-built image processing functions and provides a $\mathrm{C}$ interpreter and compiler enabling the incorporation of user-generated functions. Consequently, it provides an image processing platform that can be tailored to the needs of specific applications.

\section{Selection of Angiograms}

Angiograms obtained on patients being investigated for age-related macular degeneration (ARMD) by scanning laser fluorescein angiography in the Retinal Photography Service of Ninewells Hospital between August 1992 and December 1993 were reviewed. All patients had standard angiography. Prior to the angiograms the fundus is imaged with the SLO with the argon green laser and the images recorded. Three millilitres of $20 \%$ sodium fluorescein are injected via the anticubital vein, rapidly followed by injection of $3 \mathrm{ml}$ saline. The fluorescein angiogram was recorded using the argon blue laser. Late images were recorded at 5 minutes. Angiograms which were not continuous to 1 minute due to an interruption in recording (usually because the other eye had been imaged) were excluded.

Eighty-six angiograms which were judged to be of good quality were analysed and categorised as follows: dry maculopathy (i.e. atrophic pigment clumping and drusen), geographic atrophy of the retinal pigment epithelium, retinal pigment epithelial detachment (PED) and classical subretinal neovascularisation (SRNV). Classical SRNV demonstrated either a vascular pattern (net, seafan or cartwheel) or a single or multiple focus of progressively increasing fluorescence with leakage. Occult SRNV was excluded. The diagnosis of the various forms of ARMD above was based on currently recognised angiographic criteria. Retinal pigment epithelial atrophy and drusen were not grouped separately as it proved impossible to distinguish the two groups clearly by the retrospective analysis of angiograms. Some drusen behave similarly to retinal pigment epithelial defects angiographically with typical 
'window defects'. Both were categorised as 'dry maculopathy'.

\section{Image Acquisition}

An important prerequisite for the quantitation of fluorescein dynamics is the presence of a calibrated and reliable time axis. That is, one must be able to determine at exactly which point in the angiogram frames were recorded. One method of defining the start of the sequence would be to use the time of injection. However, this approach suffers from the drawback that arm-to-retina time depends on many variable factors related either to patient characteristics or the integrity of the injection. A more robust method is to look for dye arrival in the field of view itself. In this work, the onset of fluorescence was defined as the point at which the mean grey level in two consecutive frames increased by $20 \%$ and $30 \%$ respectively over the minimum mean grey level measured up to that point. An example is given in Fig. 2. The use of two consecutive frames provided discrimination against random variations in the mean grey level. This methodology proved highly reliable in practice.

For the purposes of this study frames were captured at the onset of fluorescence, thence at 5 second intervals until 1 minute had elapsed; a final late image was captured at 5 minutes. Of course, faster acquisition rates are possible and limited only by the speed of the digitiser board and the time required to move the video image from the buffer of the grabber board to the computer memory. Currently the minimum time with our system is 1.5-2.2 seconds between digitised frames. Selection of images is necessary to reduce computer memory requirement. Continuous retrieval of images at 25 frames per second would require immense data storage capacity. For the purposes of this study it was decided that 12 images (11 images in the first minute of dye arrival and a late image at 5 minutes) would provide sufficient information (Fig. 3).

\section{Image Registration}

Image registration was achieved using an algorithm designed specifically for the purpose, full details of which have been described by Noack and Sutton. ${ }^{8}$ Briefly, the algorithm is designed to perform automated registration of fluorescein angiograms and is based on localised correlation of binary templates in binary thresholded gradient images. Typical placement of the template is shown in Fig. 4, which illustrates two sequential images. The use of this algorithm decreases the calculation time by up to 180 times when compared with the fastest applicable algorithm found in the literature. The algorithm detects only translational movement of images but proves to be robust against rotational movement from small angles $\left(<4^{\circ}\right)$ encountered in ophthalmic imaging. In order to accommodate the inevitable fact that sequential frames are not carbon copies of each other, the algorithm has a built-in variable match quality, usually set at $70 \%$. Registration accuracy is estimated to be \pm 2 pixels. The performance of the algorithm was found to compare favourably with previously described algorithms. ${ }^{9.11}$

\section{Image Quantitation}

Angiograms from each of the patients included in the study were analysed by first registering the image sequences and then determining fluorescence as a function of time in the area of maximum fluorescence. Fluorescence in a control area devoid of retinal blood vessels and any obvious pathology was taken as an indicator of the background fluorescence.

Cases were studied to determine the most reliable position to take the background readings from; it was found that the area temporal to the macula was least subject to variation. This area was therefore chosen as the area from which background readings were taken in all cases studied.

In each case a rectangular image analysis window (region of interest) was positioned over the point of maximum fluorescence of the area/lesion being considered, with the proviso that it was contained entirely within the area of fluorescence (Fig. 5). Depending on the size of the lesion, the size of the region of interest was adjusted to encompass the maximum area possible. This varied from a small area (e.g. drusen) to a large area (e.g. in some retinal pigment epithelial detachments). A region of interest of exactly the same size was also positioned in the area temporal to the macula. The mean intensity in both the lesion and background regions of interest was then determined from each image in the registered sequence.

Fluorescence versus time curves from the area of maximal fluorescence and background were then constructed for each case being analysed. The ratio of the two curves was calculated and plotted. The rationale behind this approach was that it eliminates errors resulting from variation in overall fluorescence of the whole image. Such errors could result from a number of factors such as eye movement or the incident light being partially blocked by the pupil margin. Taking the ratio also facilitates comparisons between patients, since the variability introduced by differing injection techniques will in theory be eliminated.

\section{RESULTS}

Of the 86 scanning laser fluorescein angiograms studied, 39 were suitable for analysis by this technique. Forty-five angiograms were excluded because 4 or more of the 12 frames grabbed 


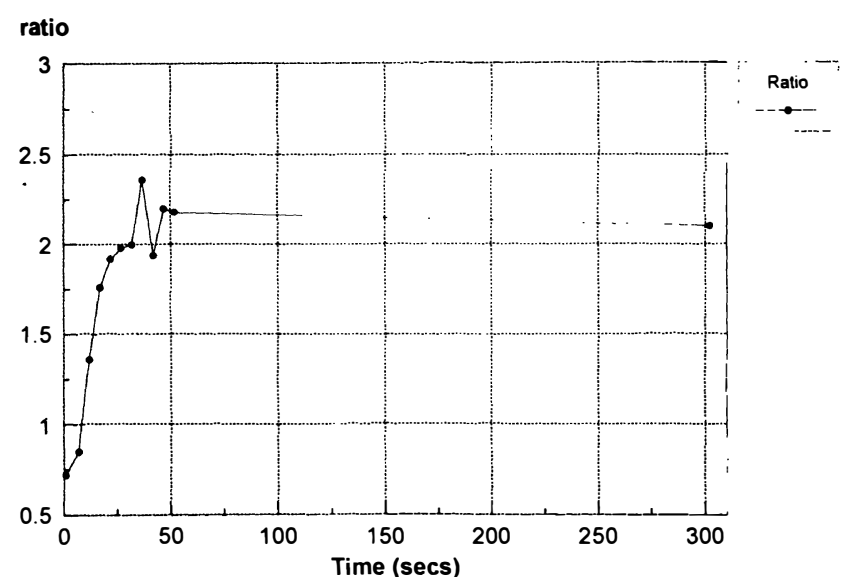

Fig. 6. A typical plot of fluorescence versus time in $S R N V$.

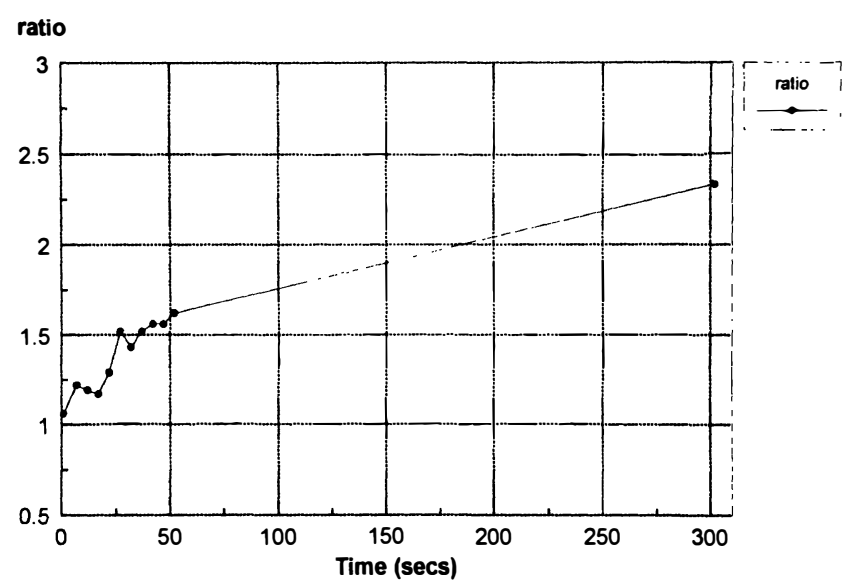

Fig. 7. A typical plot of fluorescence versus time in a case of PED.

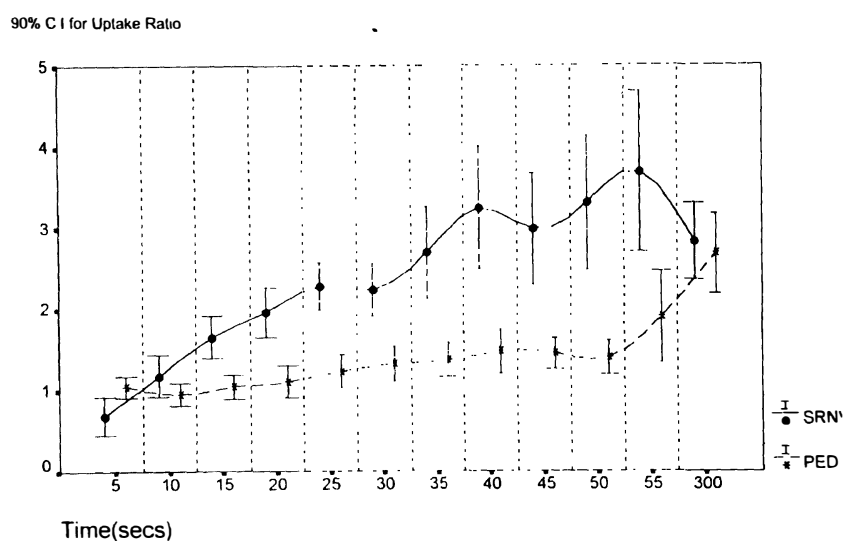

Fig. 8. Mean fluorescence uptake for SRNV and PED plotted with time, illustrating a $90 \%$ confidence limit of the mean.

occurred during a blink. A further 2 cases were excluded because the registration algorithm failed to work correctly. The final cohort included 17 cases of classical SRNV (mean age 70 years), 7 of PED (mean age 70 years), 4 cases of geographic atrophy (mean age 82 years) and 11 dry maculopathies (mean age 74 years).

There was a definite pattern associated with the fluorescence characteristics of both SRNV and PED. A typical plot of fluorescein/background ratio versus time for SRNV is shown in Fig. 6. It will be observed that there is a rapid increase in fluorescence through the first minute followed by a slow decline through to 5 minutes after fluorescein arrival. All cases of SRNV conformed to this pattern. with the time of the maximum varying between 30 and 50 seconds. Fig. 7 shows a typical plot of fluorescein/background ratio versus time for a PED patient; in this case it can be seen that the uptake ratio gradually increases from time zero, reaching a maximum at the time of the late image. To demonstrate the overall pattern in a relatively objective manner, the mean fluorescein/ background ratios for all 17 cases of SRNV and the 7 cases of PED were plotted against time as illustrated in Fig. 8. Also shown in Fig. 8, as error bars, are the $90 \%$ confidence limits of this mean. It will be seen that the maximum value of fluorescence of SRNV is approximately 3.5 times the background level.

The group classified as dry maculopathy showed marked variation in fluorescence with time and no consistent pattern was demonstrated. This was the case with readings over both drusen and pigment epithelial atrophy. Some cases showed early fluorescence with a progressive decline in fluorescence and others showed increased late fluorescence. The peak fluorescence was variable in intensity and time from dye arrival. Geographic atrophy showed the most inconsistent results, with widely varying readings and variable patterns.

\section{DISCUSSION}

The fluorescein angiographic characteristics of various forms of ARMD are well described. ${ }^{11}$ In clinical practice the analysis of angiograms is based on the subjective judgement of the ophthalmologist in relation to spatial and temporal distribution of hyperfluorescence. Several lesions, including retinal pigment epithelial defects, drusen, retinal pigment epithelial detachment and CRNV, all cause hyperfluorescence and have recognised fluorescein angiographic features. Many patients have ill-defined or occult CRNV. A reliable method of quantifying fluorescence is desirable as it may help define the characteristics of these lesions more accurately and provide reliable information to assist analysis of the natural history, and response of the disease to treatment. This study describes a method of quantitation of fluorescence which employs a novel method of image acquisition and registration that is worthy of further development.

To study sequential fluorescein dynamics quantitatively it is necessary to locate the area from which measurements are taken exactly, and there has to be correction for eye movements which occur between different images (i.e. image registration). Techniques 
employed using photographic images are laborious and unreliable. They usually involve each frame to be analysed being superimposed on a template and measurements being taken from a standard grid. Measurements of fluorescence involve densitometry methods. To analyse digital images a number of approaches have been reported in an effort to solve the problem of image registration. Cross-correlation between two complete frames has been employed. ${ }^{12,13}$ Others used features based on registration of the blood vessels (selected by operator control). A more recent approach is the use of binary templates in threshold images. ${ }^{14,15}$ This study employed a localised binary correlation previously described by Noack and Sutton. ${ }^{8}$ This is a complex though efficient algorithm and was found to compare favourably with previously described algorithms. ${ }^{9,10}$

A number of problems were identified in this study which were related to the method and the retrospective analysis of angiograms. The image acquisition was dependent on a number of factors and a large number of angiograms were excluded from study. A vast amount of information is present on each image and as computer memory is restricted we had to limit the number of images selected. The SLO gathers images at 25 frames per second; we acquired images every 5 seconds and excluded cases where 4 or more images were lost in the first minute. Poorquality images were mainly due to blinking in our study. The computer could in future be programmed to accept a grabbed frame only if it did not include a blink. It could alternatively grab several frames around each time selected and be programmed to choose one according to preselected criteria. It would be desirable to analyse many more images than we selected and to increase the analysis period to later frames. Further research would identify the most efficient retrieval times for useful data analysis. A large number of variables exist in the analysis of fluorescein angiograms, particularly in such a heterogeneous disease as ARMD. Our most consistent results in terms of the behaviour of hyperfluorescence with time were with SRNV and PED. It is not surprising that a more variable pattern was evident with cases of dry maculopathy. Fluorescence evident on angiograms depends on a large number of factors. Hyperfluorescence may be attributable to drusen, pigment epithelial atrophy, changes in Bruch's membrane, abnormal blood vessels with leakage or pooling of fluorescein. The fluorescence characteristics of the various forms of drusen differ and the choriocapillaris may be atrophic or relatively normal. Variations in choroidal perfusion pattern have been described in ARMD ${ }^{16-18}$ and cases of gradual ooze of fluorescein from the retinal pigment epithelium have been described. ${ }^{19}$ There are also variations in fluorescence due to pulsatile flow, recirculation of dye and injection artefacts. Hypofluorescence may occur due to abnormal vascular filling or blocked fluorescence by retinal pigment epithelial hyperplasia, turbid subretinal fluid, exudate or blood.

We found we could not reliably differentiate drusen from pigment epithelial atrophy by retrospective analysis of angiograms. In many angiograms there is a multiplicity of hyperfluorescent lesions and to say that one lesion was a drusen and another a window defect was not possible. The method described is therefore not useful in assessing drusen and pigment epithelial atrophy and it is probably not appropriate to group both together. Prospective study may define these groups more clearly. It is also likely that analysis of factors other than the degree of fluorescence have more diagnostic significance. Image analysis of the number and pattern of fluorescent features on the image, for example, may prove more useful. Despite these factors the behaviour of fluorescein leakage from SRNV and fluorescein pooling within PED was remarkably consistent, and with further study it is hoped that these would be more clearly characterised.

Age-related macular degeneration is the major cause of blindness ${ }^{9,20-22}$ and a number of treatments other than laser therapy for SRNV have recently been proposed. A method of quantifying the angiographic features is highly desirable. Although this study is preliminary, it demonstrates a reliable though rudimentary method of quantitation which offers a solution to the significant problems of image acquisition and registration. Further development of this technique analysing various time parameters should identify the optimum images which may be of most clinical value. In this study the intensity of fluorescence was measured. There are more complex image analysis techniques which may be employed. For example, the use of region growing to count features automatically, the use of morphological operators automatically to define areas of the same type (e.g. drusen) and the use of texture analysis may permit classification of different pathologies. Image analysis techniques may be used in measuring the size of lesions, counting the number of lesions, and assessing boundary characteristics of lesions and the dynamic flow parameters of blood vessels. In relation to indocyanine green angiography, fluorescence quantitation may improve our understanding of the diagnostic features of SRNV. ${ }^{23,24}$

The authors are indebted to the Speed Pollock Memorial Research Trust for their support in this project and thank Mrs L. Rose for typing the manuscript.

Key words: Age-related macular degeneration, Fluorescein angiography, Image analysis, Image registration, Pigment epithelial detachment, Scanning laser, Subretinal neovascularisation. 


\section{REFERENCES}

1. Elsner AE. Weiter JJ. Jalkh AE. New devices for retinal imaging and functional evaluation. In Freeman R. editor. Practical atlas of retinal discase and therapy. New York: Raven Press. 1993:19-35.

2. Webb RH. Hughes (jW. Pomerantzeff (). Flying spot TV ophthalmoscope. Appl Optics 198():19:2991-7.

3. Webb RH. Hughes (iW. Delori FC. Confocal scanning laser ophthalmoscope. Appl Optics 1987:26:1492-9.

4. Woon WH. Fitzke FW. Choler (iH. Greenwood (jD. Marshall J. The scanning laser ophthalmoscope: basic principles and applications. J Ophthalmic Photography 199():12:17-23.

5. (jonzale\% RC: Wintz. P. Digital image processing. 2nd ed. Reading. MA: Addison-Wesley. 1987.

6. Woll S. et al. Scanning laser ophthalmoscopy for the quantitation of retinal blood-flow parameters: a new imaging technique. In Elsner et al. Scanning laser topography. Munich: Quintessenz. 199().

7. Manivannan A. Sharp PF. Phillips RP. Forrester JV. Digital fundus imaging using a scanning laser ophthalmoscope. Physiol Meas 1993:14:43-56.

8. Noack J, Sutton DC. An algorithm for the fast registration of image sequences obtained with a scanning laser ophthalmoscope. Phys Med Biol 1994: $39: 9(1) 7-15$.

9. Worson DP, Hughes (jW. Webb RH. Fundus tracking with the scanning laser ophthalmoscope. Appl Opt 199():26:15()()-4.

10. ()tt D. Lades M. Holtholf K. Eckmillar R. A general numerical method evaluating three-dimensional eve rotations by scanning laser ophthalmoscopy. Ophthalmic Physiol (Opt 199():1():286-9).

11. Bressler NM. Bressler SB. Fine SL. Age-related macular degeneration. Surv Ophthalmol 1988:32: $37.3-41.3$.

12. Nagin P. Measurement of fluorescein in angiograms of the optic disc and retina using computerised image analysis. Ophthalmology 1987:92:547-52.

13. Parker JA. 't al. Measurement of torsion from multitemporal images of the eve using digital signal processing techniques. IEEE Trans Biomed Eng 1987:6:18-36.
14. Bantel T, et al. Global tracking of the ocular fundus pattern imaged by scanning laser ophthalmology. Int J Biomed Comp 1990;27:59-69.

15. Klein GJ, Baumgartner RH, Flower RW. An image processing approach to choroidal blood flow. Invest Ophthalmol Vis Sci 1990;31:629-37.

16. Piguet B, Palmvang IB, Chisholm IH, Minassian D, Bird AC. Evolution of age-related macular degeneration with choroidal perfusion abnormality. Am J Ophthalmol 1992;113:657-63.

17. Scheider A, Neuhauser L. Fluorescence characteristics of drusen during indocyanine green angiography and their possible correlation with choroidal perfusion. Ger J Ophthalmol 1992;1:328-34.

18. Staurenghi G, Bottoni F, Lonati C, Autelitano A, Orzalesi N. Drusen and choroidal filling defects: a cross sectional survey. Ophthalmologica 1992;205:178-86.

19. Boldt HO, Folk JC. Slow leakage from the retinal pigment epithelium (ooze) in age-related macular degeneration. Retina 1990;10:244-50.

2(). Kahn HA, Moorhead HB. Statistics on blindness in the model reporting areas 1969-1970. United States Department of Health Education and Welfare publication no. (NIH) 73-427. Washington, DC: US Government Printing Office, 1973

21. Leibowitz H, Krueger DE, Maunder LR, et al. The Framingham Eye Study monograph: an ophthalmological and epidemiological study of cataract, glaucoma, diabetic retinopathy, macular degeneration, and visual acuity in a general population of 2631 adults, 1973-75. Surv Ophthalmol 1980;24 (Suppl):335-620.

22. Sorsby A. The incidence of blindness in England and Wales 1963-1968. Ministry of Health Reports on Public Health and Medical Subjects No. 128. London: Her Majesty's Stationery Office, 1972.

23. Destro M, Puliafito CA. Indocyanine green videoangiography of choroidal neovascularisation. Ophthalmology 1989;96:846-52.

24. Scneider A, Voeth A, Kaboth A, Neuhauser L. Fluorescence characteristics of indocyanine green in normal choroid and in subretinal neovascular membranes. Ger J Ophthalmol 1992;1:7-11. 